\title{
Finite element analysis of plasma dust-acoustic waves
}

\author{
P. Areias ${ }^{\mathrm{a}, \mathrm{c}, \stackrel{*}{ }, \text { J.N. Sikta }}{ }^{\mathrm{a}, \mathrm{b}}$, M.P. dos Santos ${ }^{\mathrm{a}}$ \\ ${ }^{a}$ Department of Physics, University of Évora, Colégio Luís António Verney, Rua Romão Ramalho, 59, 7002-554, Évora, Portugal \\ ${ }^{\mathrm{b}}$ Department of Physics, Jahangirnagar University, 1342 Savar, Dhaka, Bangladesh \\ c CERIS/Instituto Superior Técnico, University of Lisbon, Portugal
}

\section{A R T I C L E I N F O}

\section{Keywords:}

Dusty plasma

Acoustic waves

Vortex

Finite elements

Nonlinear fluid

\begin{abstract}
A B S T R A C T
For dust acoustic solitary waves, we propose a finite element formulation of the fluid dusty plasma equations. To solve this continuum problem, a Petrov-Galerkin weak form with upwinding is applied. We consider an unmagnetized dusty plasma with negatively charged dust and Boltzmann distributions for electrons and ions. Nonlinearity of ion and electron number density as functions of the electrostatic potential is included. A fullyimplicit time-integration is used (backward-Euler method) which requires the derivative of the weak form. A three-field formulation is introduced, with dust number-density, electrostatic potential and dust velocity being the unknown fields. We test the formulation with two numerical (2D and 3D) examples where convergence with mesh size is assessed. These establish the new formulation as a predictive tool in dusty plasmas.
\end{abstract}

\section{Introduction}

In the 1920's, Irving Langmuir [1] proposed that electrons, ions and neutrals in an ionized gas can be, as a whole, considered a corpuscular material, he titled as plasma. It is now accepted that more than $99 \%$ of the known universe, in which the dust is a omnipresent ingredient, is in the plasma state $[2,3]$. Plasmas containing dust particles are important in the study of the space environment, such as asteroid zones, planetary rings (viz. Saturn rings [4]), comet tails, as well as Earth's magnetosphere [5]. In addition, dusty plasmas are observed in laboratory within Q-machines, DC discharges, and RF discharges. Dusty plasmas typically contain dust grains of micrometre or sub-micrometre size which are negatively charged by field emission, ultra-violet ray irradiation, and plasma currents [6,7]. Collective effects in micro-plasmas have been studied by Verheest [8] using many-fluid models.

The presence of grains of dust alters existing plasma wave spectra and introduces dust-acoustic waves, dust ion-acoustic waves, dust lattice waves etc. [9]. In dust acoustic waves, inertia is provided by dust particle mass and the restoring force is provided by the pressures of electrons and ions. Dust acoustic waves predicted by Rao, Shukla and Yu have been experimentally observed by Barkan et al. [10]. As mentioned by Merlino and Goree [11], in a dust acoustic wave neighbor, dust fluid elements are coupled by the electric field associated with the wave rather than by collisions, as they would be in a neutral gas.

We are not aware of antecedent finite element solutions of dusty plasma. However, two-fluid finite element solutions of plasmas exist, one significant solution has been developed by C.R. Sovinec's group, cf. $[12,13]$ using classical (continuous) Galerkin methods. Inherent instabilities caused by the convective term are dealt at the time-integration level, cf. [13]. In the work of Jardin, Breslau and Ferraro [14], the Clough-Tocher $\mathscr{C}^{1}$ triangular element was used to solve smooth magneto-hydrodynamic problems. Other solutions exist for magnetohydrodynamics, cf. $[15,16]$, which, although related to this work, are a parallel development. If the analysis involves shock waves, discontinuous Galerkin methods (cf. [17]) have been used with success with plasmas, see Levy, Shu and Yan [18].

The main goal of this first work is to create a computational framework, with a set of benchmarks, for the analysis of dusty plasma dynamics. More ingredients (magnetic field, Newton viscosity, non-Newtonian effects) will be introduced as found relevant, on top of thoroughly tested software.

Herein, we assume that dust particles have constant mass and are point charges. In addition, we consider a three component plasma consisting of electrons and ions having Boltzmann density distributions with temperatures $T_{e}$ and $T_{i}$, respectively, as well as negatively charged, heavy dust particles. To simplify the treatment, thermal motion of dust is not included. This case has been identified as "cold dust" by Rao, Shukla and $\mathrm{Yu}$ [7]. In dust-free electron-ion plasmas, ions charge generally remains constant. However, in a dusty plasma, the charge of a particle does not remain constant, cf. [11]. With the goal of obtaining a stable solution of a shock-free problem, we opt for an implicitly integrated Petrov-Galerkin formulation, which results in a very simple but effec-

\footnotetext{
* Corresponding author. Department of Physics, University of Évora, Colégio Luís António Verney, Rua Romão Ramalho, 59, 7002-554, Évora, Portugal.

E-mail address: pmaa@uevora.pt (P. Areias).
} 
Table 1

Relevant quantities and constants (cf. 5-15).

\begin{tabular}{ll}
\hline$e$ & Electron charge $\left[1.6022 \times 10^{-19} \mathrm{C}\right]$ \\
$\kappa_{B}$ & Boltzmann constant $\left[1.38065 \times 10^{-23} \mathrm{~m}^{2} \mathrm{~kg} \mathrm{~s}^{-2} \mathrm{~K}^{-1}\right]$ \\
$\varepsilon_{0}$ & Vacuum permittivity $\left[8.85419 \times 10^{-12} \mathrm{Fm}^{-1}\right]$ \\
$Z_{d}$ & Dust charge number $Z_{d}=-Q_{d} / e$ \\
$n_{d 0}$ & Dust number density at equilibrium $\left[\mathrm{m}^{-3}\right]$ \\
$n_{i 0}$ & Ion number density at equilibrium $\left[\mathrm{m}^{-3}\right]$. Approximation: $n_{i 0} \cong Z_{d} n_{d 0}$ \\
$n_{e 0}$ & Electron number density at equilibrium $\left[\mathrm{m}^{-3}\right]$. Approximation: $n_{e 0} \cong n_{i 0}$ \\
$T_{e}$ & Electron temperature $[\mathrm{K}]$ \\
$T_{i}$ & Ion temperature $[\mathrm{K}]$ \\
$Z_{i}$ & Ion charge number $Z_{i}=Q_{i} / e$ \\
$m_{d}$ & Mass of a dust particle $[\mathrm{Kg}]$ \\
$\boldsymbol{u}_{0}(\boldsymbol{x})$ & Initial dust velocity \\
$n_{d 0}$ & Initial number density of dust \\
$\overline{\boldsymbol{u}}(t)$ & Imposed velocity at the boundary $\Gamma_{u}$ \\
$\bar{n}(t)$ & Imposed density at the boundary $\Gamma_{n}$ \\
$\bar{\varphi}(t)$ & Imposed electrostatic potential at the boundary $\Gamma_{\varphi}$ \\
$\bar{t}(t)$ & Imposed electrostatic gradient at the boundary $\Gamma_{\varphi^{\prime}}$ \\
\hline
\end{tabular}

tive numerical scheme. We organize this work as follows: in Section 2 the governing equations are presented (continuity, momentum and Poisson), with the respective initial and boundary conditions. In Section 3 , the weak form using a Petrov-Galerkin combination of test/trial functions is presented. This is followed, in Section 4, by the discretization using Streaming Upwind Petrov-Galerkin (SUPG) [19] shape functions. In Section 5, a set of 2D and 3D representative numerical examples is shown, confirming the robustness in terms of mesh and step-size effects in the numerical results. Finally, in Section 6, conclusions are drawn.

\section{Governing equations}

\subsection{Characteristic quantities}

We consider the following independent unknown fields:

- $n_{d}$ : number density of dust

- $\boldsymbol{u}_{d}$ : dust velocity

- $\varphi$ : electrostatic wave potential

Equations for a dusty plasma typically make use of normalized quantities, which in turn depend on characteristic values. We introduce the Debye length for a dusty plasma, using the ion temperature, as (see the approximation $\lambda_{d} \cong \lambda_{i}$ in Ref. [3]):

$\lambda_{i}=\frac{1}{Z_{i} e} \sqrt{\frac{\varepsilon_{0} \kappa_{B} T_{i}}{n_{i}}}$

where $\varepsilon_{0}$ is the electric permittivity of free space, $\kappa_{B}$ is the Boltzmann constant, $T_{i}$ is the ion temperature (with Kelvin units), $Z_{i}$ is the ion charge number, $n_{i}$ is the number density of ions and $e$ is the electron charge. In this work, ions are positively charged and dust is negatively charged. In addition, $\lambda_{i}$ serves as a characteristic length-scale. In terms of time, we introduce thermal speed of dust, $v_{d}$, which is obtained from the dust temperature $T_{d}$ and the dust particle mass $m_{d}$ as:

$v_{d}=\sqrt{\frac{\kappa_{B} T_{d}}{m_{d}}}$

The dust acoustic speed $\left(c_{d a}\right)$ for cold dust is given by (cf. [9]):

$c_{d a}=\sqrt{\frac{Z_{d} \kappa_{B} T_{i}}{m_{d}}}$

where $Z_{d}$ is the dust charge number. Making use of equilibrium (initial) quasi-neutrality, we have:

$n_{i 0} Z_{i}=n_{e 0}+n_{d 0} Z_{d}$

Table 2
Properties in use for the numerical
examples (ionosphere [25]).
\begin{tabular}{ll}
\hline$Z_{d}$ & 1000 \\
$n_{d 0}$ & $1 \times 10^{8} \mathrm{~m}^{-3}$ \\
$n_{i 0}$ & $3 \times 10^{11} \mathrm{~m}^{-3}$ \\
$n_{e 0}$ & $2 \times 10^{11} \mathrm{~m}^{-3}$ \\
$T_{e}$ & $1160.45 \mathrm{~K}$ \\
$T_{i}$ & $1160.45 \mathrm{~K}$ \\
$m_{d}$ & $1.05893 \times 10^{-12} \mathrm{~kg}$ \\
$\lambda_{e}$ & $0.00429109 \mathrm{~m}$ \\
$\lambda_{i}$ & $0.00429109 \mathrm{~m}$ \\
$T^{d}$ & $6.93282 \mathrm{~s}$ \\
$\varphi^{\star}$ & $0.0999597 \mathrm{NmC}^{-1}$
\end{tabular}

where $Z_{i}=1$ considered in the remainder of this work. In (4), $n_{i 0}, n_{e 0}$ and $n_{d 0}$ are the ion, electron and dust number densities for $t=0$, which is identified as equilibrium time.

2.2. Fluid theory of the dust acoustic wave: differential equations and boundary conditions

Dust is considered cold, $T_{d} \ll T_{i}$ in the following equations. The domain under consideration is denoted by $\Omega$ and the time interval under consideration is $[0, T]$. The governing equations for these unknown fields are, given that $x \in \Omega$ and $t \in[0, T]$,

Continuity equation on $\Omega \times[0, \mathrm{~T}]$ :

$\dot{n}_{d}+\nabla \cdot\left(n_{d} u_{d}\right)=0$

Momentum equation on $\Omega \times[0, \mathrm{~T}]$ :

$n_{d} m_{d} \dot{\boldsymbol{u}}_{d}+n_{d} m_{d}\left(\nabla \boldsymbol{u}_{d}\right) \cdot \boldsymbol{u}_{d}+m_{d} c_{d a}^{2} \nabla n_{d}-n_{d} e Z_{d} \nabla \varphi=0$

Poisson-like equation on $\Omega \times[0, \mathrm{~T}]$ :

$\nabla^{2} \varphi+\frac{e}{\varepsilon_{0}}\left(n_{i}-n_{e}-n_{d} Z_{d}\right)=0$

see Ref. [7]. These equations are complemented by the initial and boundary conditions for the unknown functions $\boldsymbol{u}_{d}(\boldsymbol{x}, t), n_{d}(\boldsymbol{x}, t)$ and $\varphi(\boldsymbol{x}, t)$ :

$u_{d}(x, 0)=u_{0}(x)$

$n_{d}(\boldsymbol{x}, 0)=n_{d 0}(\boldsymbol{x})$ 


$$
n_{d 0}^{\star}=0.5
$$

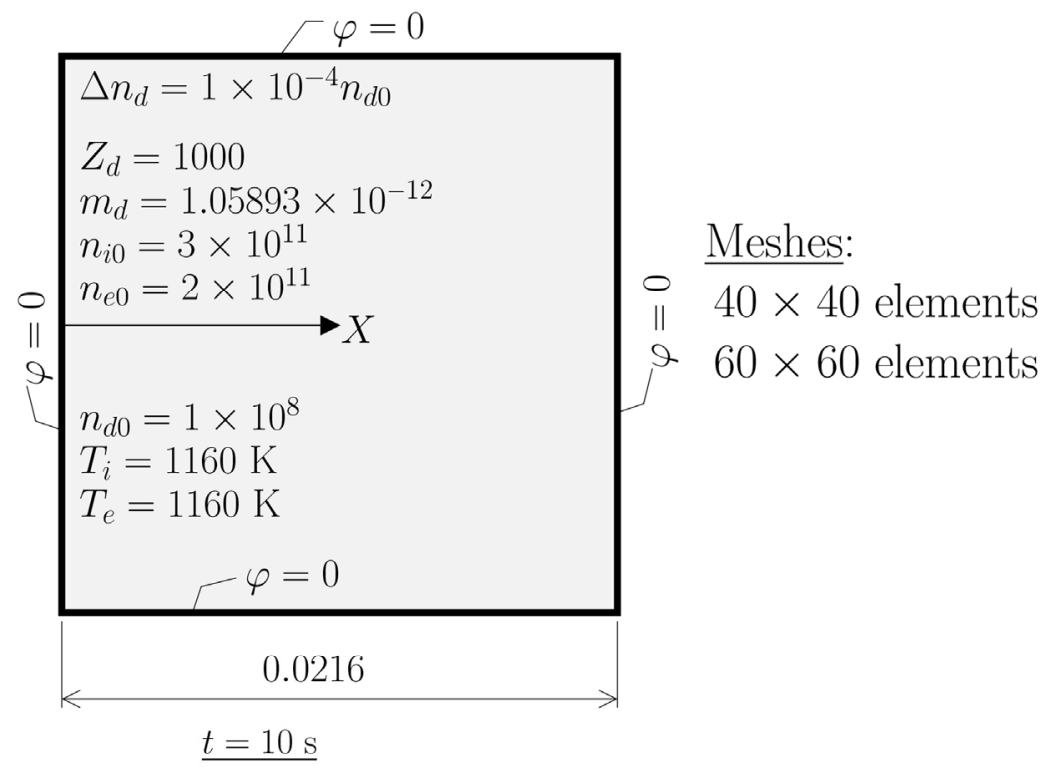

$u_{1}$

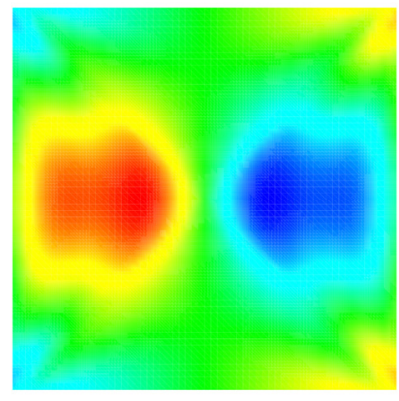

$n_{d}^{\star}$

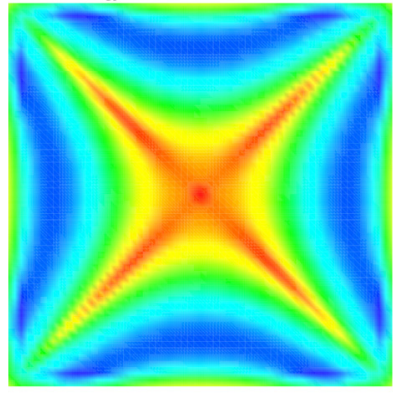

$\mathrm{c}_{e}$

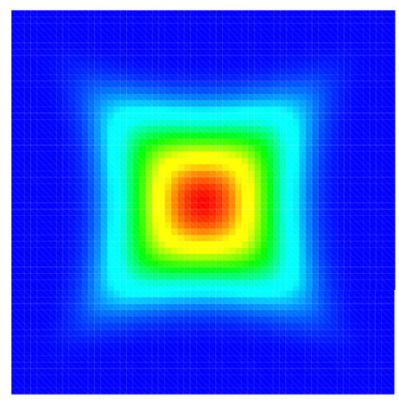

$u_{2}$
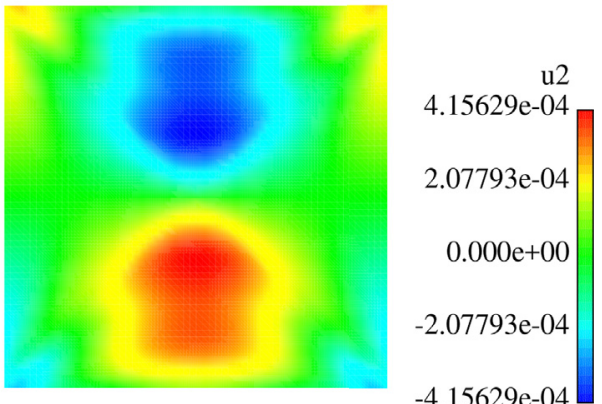

$4.15629 \mathrm{e}-04$

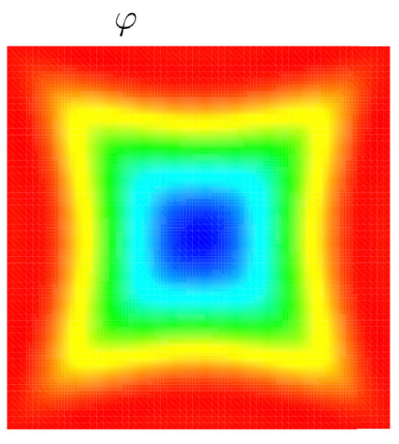

phi/phistar

$6.4703 \mathrm{e}-04$
$-1.5854 \mathrm{e}-02$
$-3.2351 \mathrm{e}-02$
$-4.8862 \mathrm{e}-02$
$-6.5353 \mathrm{e}-02$

$6.4703 \mathrm{e}-04$
$-1.5854 \mathrm{e}-02$
$-3.2351 \mathrm{e}-02$
$-4.8862 \mathrm{e}-02$
$-6.5353 \mathrm{e}-02$

$6.4703 \mathrm{e}-04$
$-1.5854 \mathrm{e}-02$
$-3.2351 \mathrm{e}-02$
$-4.8862 \mathrm{e}-02$
$-6.5353 \mathrm{e}-02$

$6.4703 \mathrm{e}-04$
$-1.5854 \mathrm{e}-02$
$-3.2351 \mathrm{e}-02$
$-4.8862 \mathrm{e}-02$
$-6.5353 \mathrm{e}-02$

$6.4703 \mathrm{e}-04$
$-1.5854 \mathrm{e}-02$
$-3.2351 \mathrm{e}-02$
$-4.8862 \mathrm{e}-02$
$-6.5353 \mathrm{e}-02$ u2

4

$c_{i}$

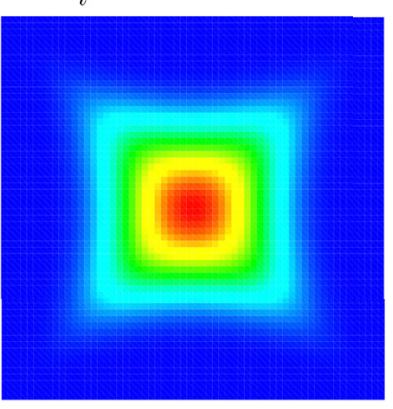

$\mathrm{ci}$

$2.034 \mathrm{e}-03$

$1.526 \mathrm{e}-03$

$1.017 \mathrm{e}-03$

$5.086 \mathrm{e}-04$

$0.000 \mathrm{e}+00$

Fig. 1. Square domain with initial charge imbalance $(t=10 \mathrm{~s})$. 


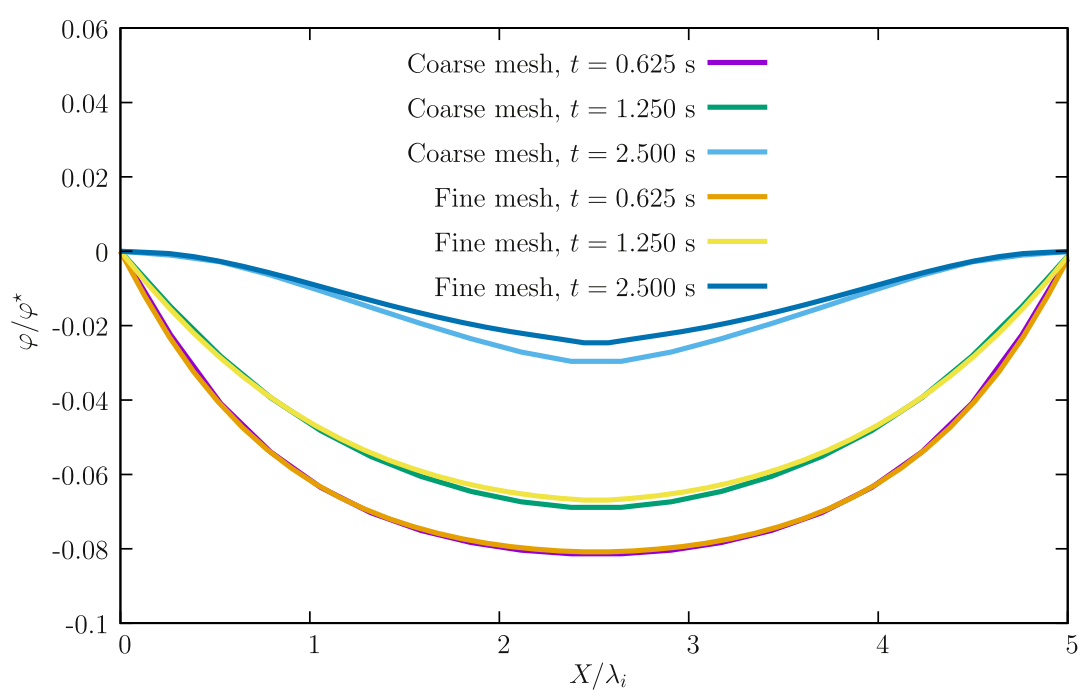

(a) Evolution of $\varphi / \varphi^{\star}$ for the two meshes

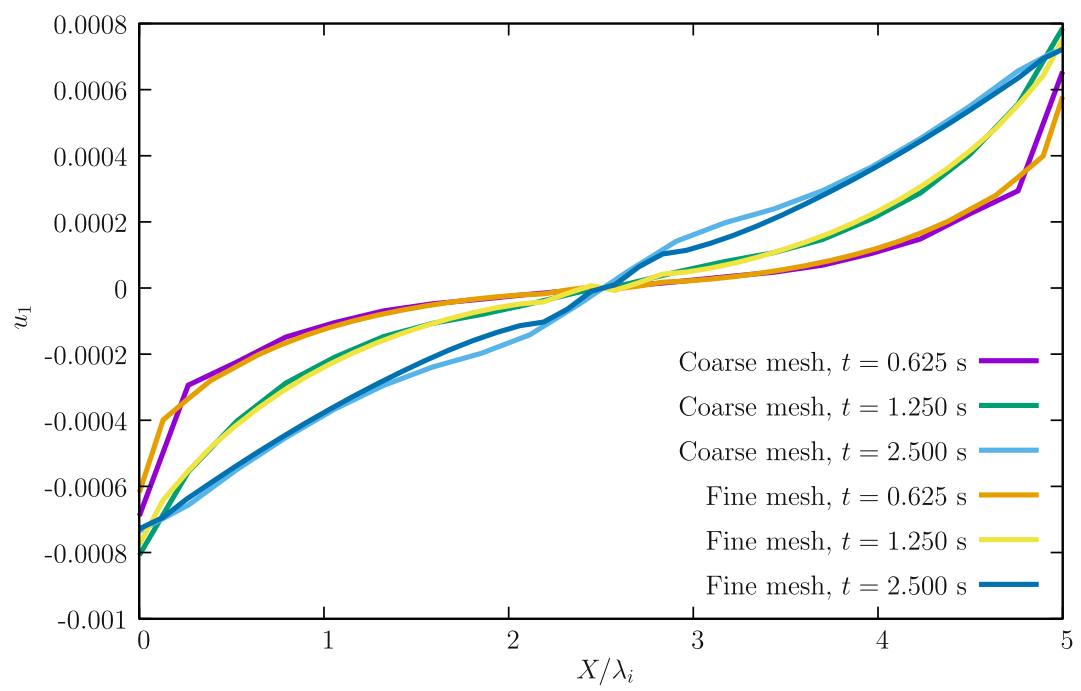

(b) Evolution of $u \equiv u_{1}$ for the two meshes

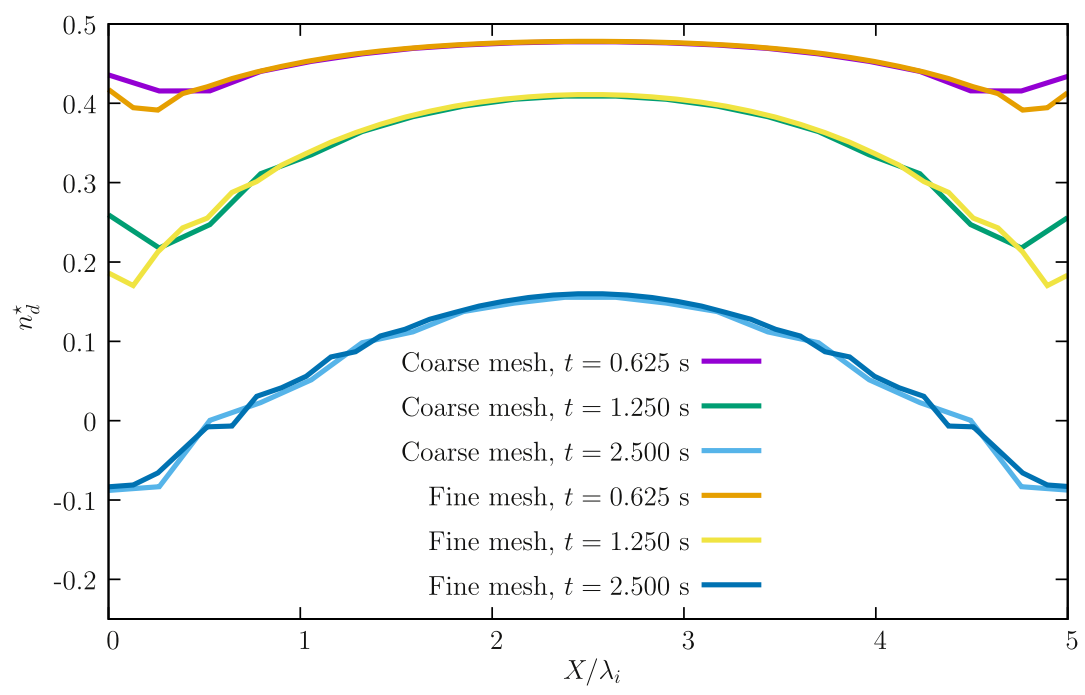

(c) Evolution of $n_{d}^{\star}$ for the two meshes

Fig. 2. Square domain with initial charge imbalance: variation of $u \equiv u_{1}, \varphi / \varphi^{\star}$ and $n_{d}^{\star}$. 

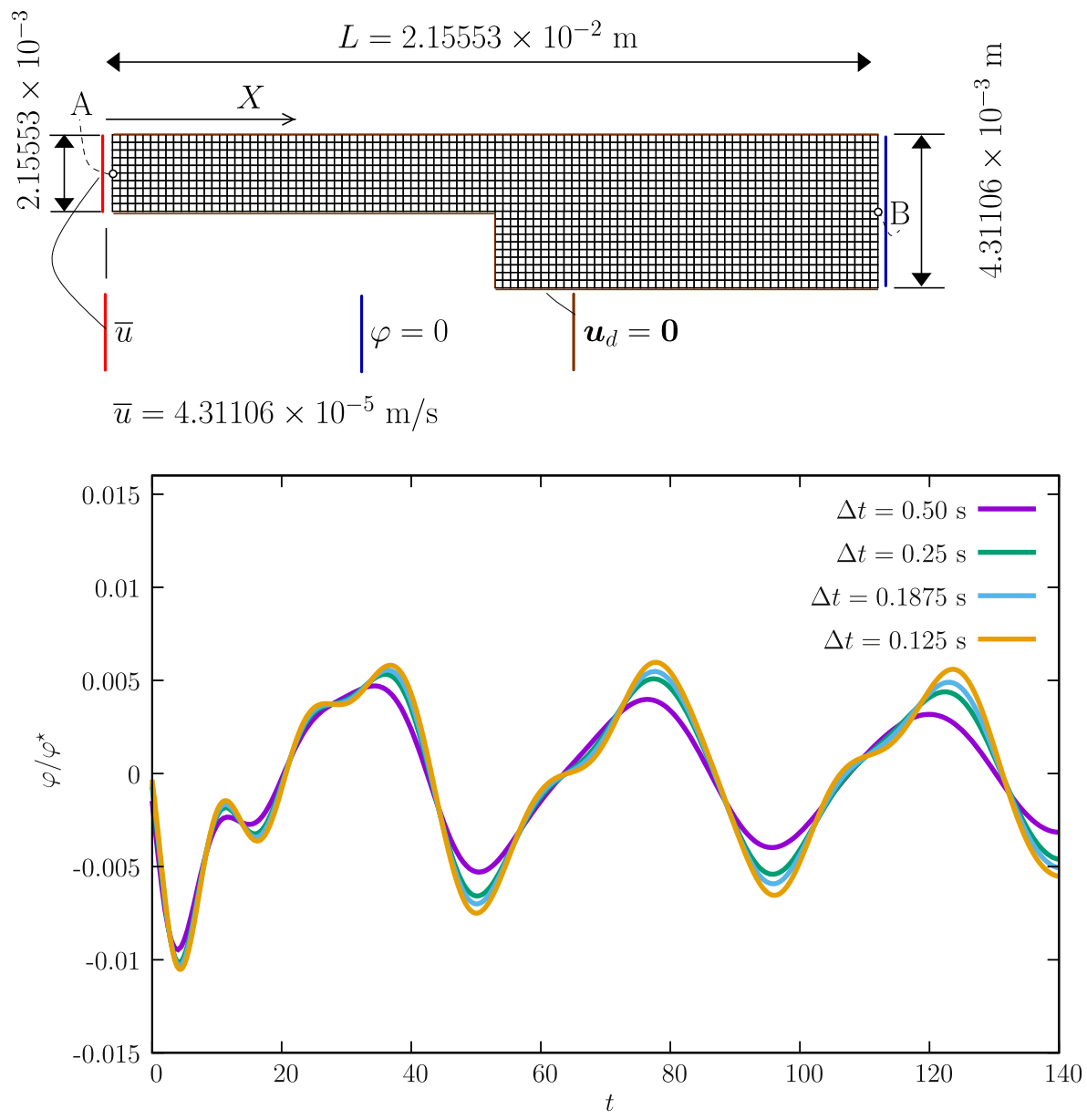

(a) Point A: evolution of $\varphi / \varphi^{\star}$ for four different values of $\Delta t$.

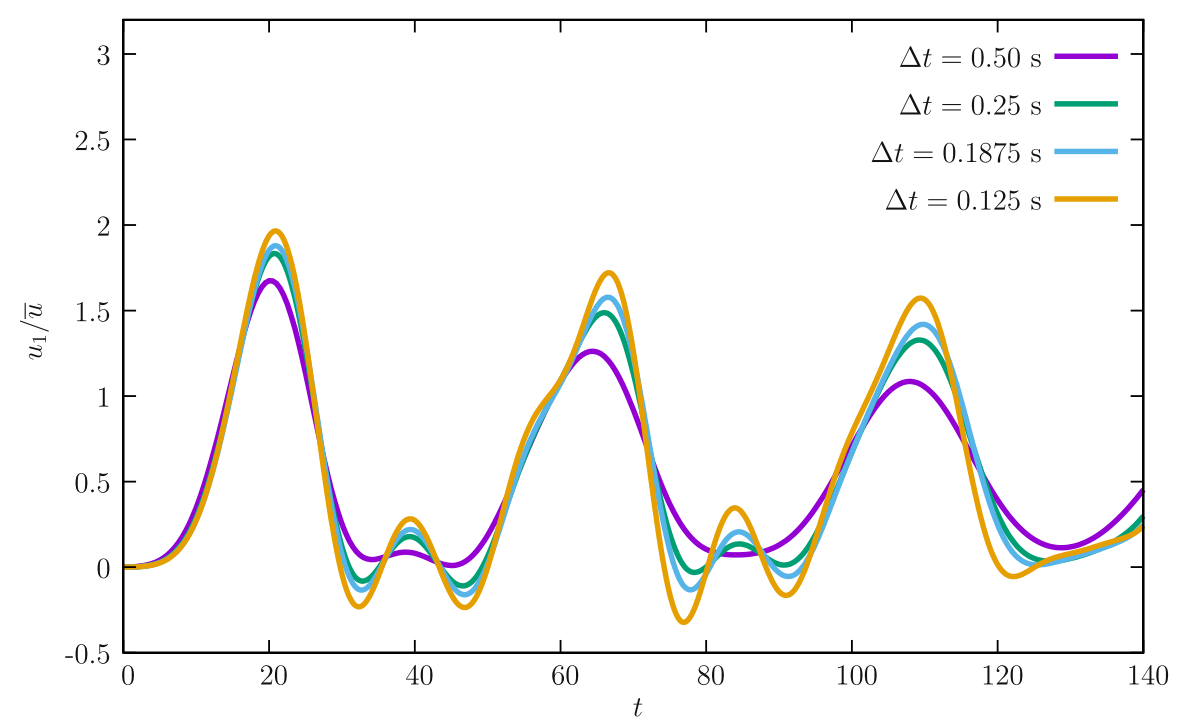

(b) Point B: evolution of $u_{1} / \bar{u}$ for four different values of $\Delta t$.

Fig. 3. Variable width slab: relevant data and results for $u_{1}$ and $\varphi$. Points A and B indicate monitored $\varphi / \varphi^{\star}$ and $u_{1}$, respectively. 


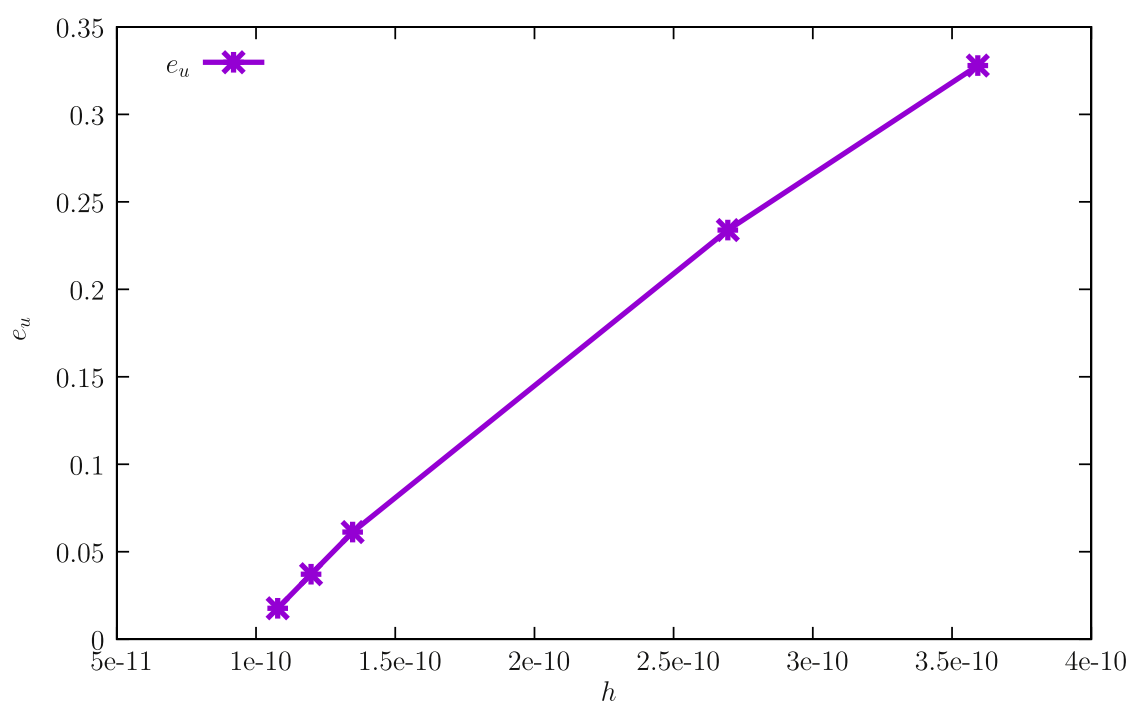

(a) $e_{u}$

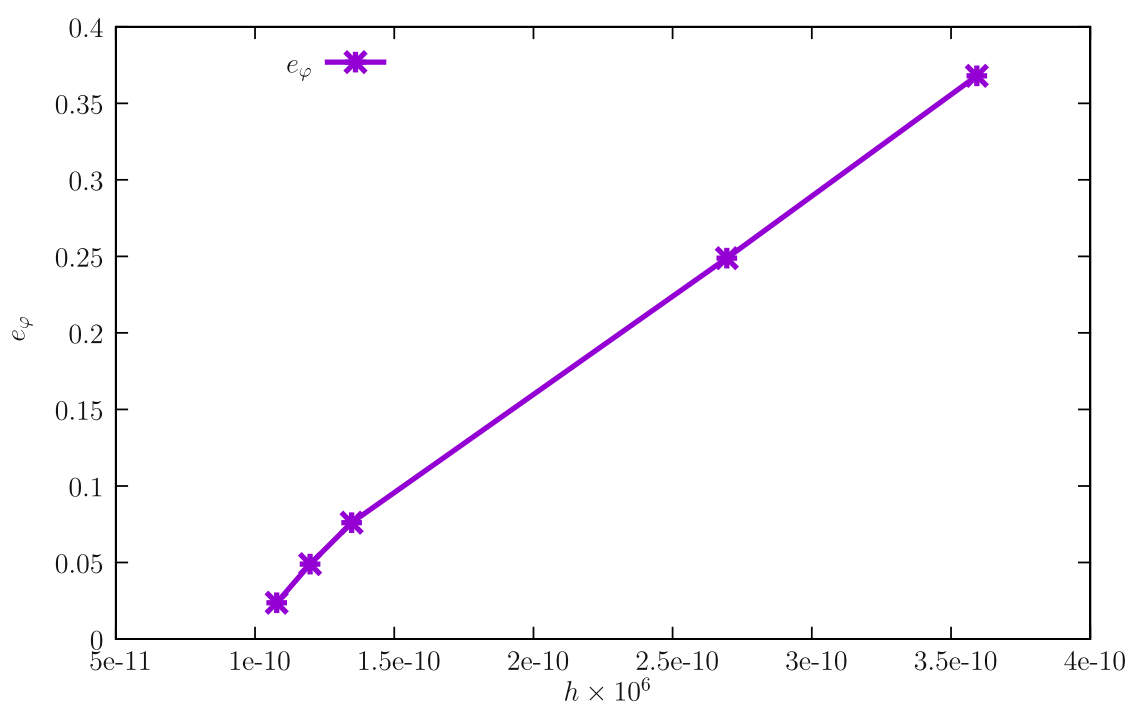

(b) $e_{\varphi}$

Fig. 4. Errors $e_{u}$ and $e_{\varphi}$ as functions of the element size $h$.

$\left.\boldsymbol{u}_{d}(\boldsymbol{x}, t)\right|_{x \in \Gamma_{u}}=\overline{\boldsymbol{u}}(t)$

$\left.n_{d}(\boldsymbol{x}, t)\right|_{x \in \Gamma_{n}}=\bar{n}(t)$

$\left.\varphi(\boldsymbol{x}, t)\right|_{x \in \Gamma_{\varphi}}=\bar{\varphi}(t)$

$\left.\boldsymbol{v} \cdot \nabla \varphi(\boldsymbol{x}, t)\right|_{\boldsymbol{x} \in \Gamma_{\varphi^{\prime}}}=\bar{t}(t)$

where $\Gamma=\partial \Omega=\Gamma_{u} \cup \Gamma_{n} \cup \Gamma_{\varphi} \cup \Gamma_{\varphi^{\prime}}$. We have $\boldsymbol{v}$ as the outer normal to $\Gamma$. In equation (7) the gradient of $\boldsymbol{u}_{d}$ makes use of the notation $\left[\nabla \boldsymbol{u}_{d}\right]_{i j}=$ $\frac{\partial u_{d i}}{\partial x_{j}}$. In (7), the electron number density $n_{e}$, and the ion number density $n_{i}$, are given by the Boltzmann distributions, corresponding to a very low frequency wave [3]:

$n_{e}=n_{e 0} \exp \left[\frac{e \varphi}{\kappa_{B} T_{e}}\right]$ $n_{i}=n_{i 0} \exp \left[-\frac{e \varphi}{\kappa_{B} T_{i}}\right]$

These relations (14-15) are constitutive equations for $n_{i}$ and $n_{e}$. It is straightforward to show that, in (14-15), the argument of the exponential is dimensionless. Characteristic lengths based on (14-15) are obtained by linearization of both expressions. For $\lambda_{e}$ we have:

$\lambda_{e}=\frac{1}{e} \sqrt{\frac{\varepsilon_{0} \kappa_{B} T_{e}}{n_{e 0}}}$

which is the Debye length for electrons. The characteristic time scale is obtained from the dust angular frequency:

$\omega_{d}=Z_{d} e \sqrt{\frac{n_{d 0}}{\varepsilon_{0} m_{d}}}$

using the period, $T^{d}=\frac{2 \pi}{\omega_{d}}$. Relevant quantities are summarized in Table 1. Unknown fields are $n_{d}, \boldsymbol{u}_{d}$ and $\varphi$. Since $n_{d}$ for $t=0$ is known to be $n_{d 0}$, we advantageously transform the continuity equation to read: 


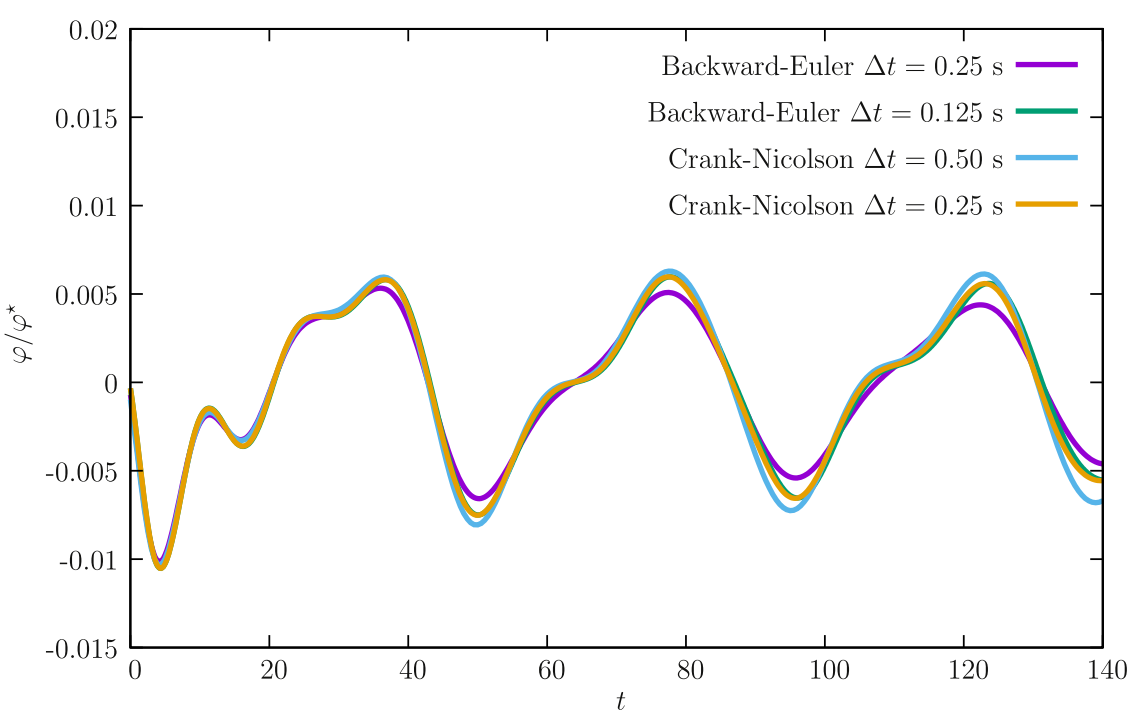

(a) Point A: evolution of $\varphi / \varphi^{\star}$ for backward-Euler and Crank-Nicolson.

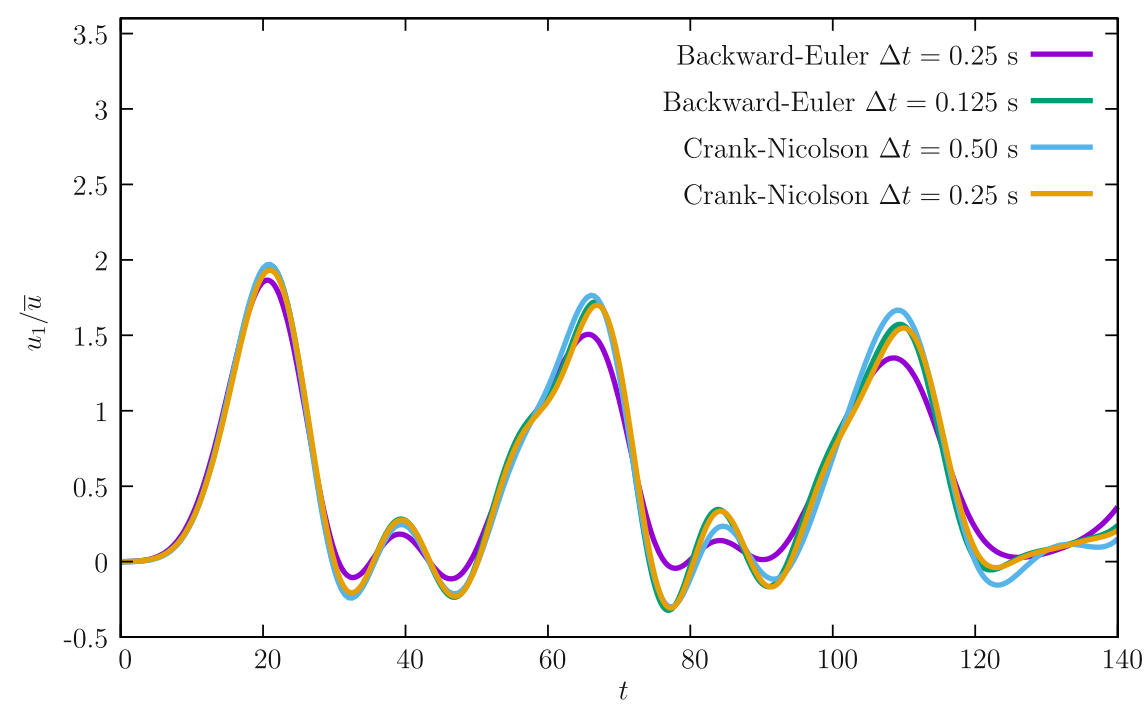

(b) Point B: evolution of $u_{1} / \bar{u}$ for backward-Euler and Crank-Nicolson.

Fig. 5. Comparison of backward-Euler and Crank-Nicolson algorithms for $\varphi / \varphi^{\star}$ and $u_{1} / u$.

$\dot{n}_{d}^{\star}+\nabla \cdot\left[\left(n_{d}^{\star}+1\right) \boldsymbol{u}_{d}\right]=0$

where

$n_{d}=\left(1+n_{d}^{\star}\right) n_{d 0}$

In equation (18) it is assumed that $n_{d 0}$ is uniform. For the Poissonlike equation, after inserting (19), we have,

$\nabla^{2} \varphi+\frac{e}{\varepsilon_{0}}\left[n_{i}-n_{e}-n_{d 0}\left(1+n_{d}^{\star}\right) Z_{d}\right]=0$

\section{Weak form with distinction of the convective term}

Numerical time-integration of equations (5-7) is performed with the backward-Euler method. In 2D, the integrated versions of (5-7) between time-steps $t_{k}$ and $t_{k+1}$ (with $\Delta t=t_{k+1}-t_{k}$ ) are straightforward:

$\left(n_{k+1}^{\star}+1\right)\left(\frac{\partial u_{1}}{\partial x_{1}}+\frac{\partial u_{2}}{\partial x_{2}}\right)+\frac{n_{k+1}^{\star}-n_{k}^{\star}}{\Delta t}+\frac{\partial n_{k+1}^{\star}}{\partial x_{1}} u_{1}+\frac{\partial n_{k+1}^{\star}}{\partial x_{2}} u_{2}=0$

$$
\begin{gathered}
\left(n_{k+1}^{\star}+1\right)\left(\frac{\boldsymbol{u}_{k+1}-\boldsymbol{u}_{k}}{\Delta t}\right)+\left.\left(n_{k+1}^{\star}+1\right)\left\{\begin{array}{l}
u_{1} \frac{\partial u_{1}}{\partial x_{1}}+u_{2} \frac{\partial u_{1}}{\partial x_{2}} \\
u_{1} \frac{\partial u_{2}}{\partial x_{1}}+u_{2} \frac{\partial u_{2}}{\partial x_{2}}
\end{array}\right\}\right|_{k+1}+ \\
-\left.\frac{e\left(n_{k+1}^{\star}+1\right) z_{d}}{m_{d}}\left\{\begin{array}{l}
\frac{\partial \varphi}{\partial x_{1}} \\
\frac{\partial \varphi}{\partial x_{2}}
\end{array}\right\}\right|_{k+1}+\left.\frac{c_{d a}^{2}}{n_{d 0}}\left\{\begin{array}{l}
\frac{\partial n^{\star}}{\partial x_{1}} \\
\frac{\partial n^{\star}}{\partial x_{2}}
\end{array}\right\}\right|_{k+1}=\left\{\begin{array}{l}
0 \\
0
\end{array}\right\} \\
\frac{\partial^{2} \varphi_{k+1}}{\partial x_{1}^{2}}+\frac{\partial^{2} \varphi_{k+1}}{\partial x_{2}^{2}}+\frac{e}{\varepsilon_{0}}\left[n_{i}-n_{e}-n_{d 0}\left(1+n_{k+1}^{\star}\right) z_{d}\right]=0
\end{gathered}
$$

To make use of finite elements, equations (21-23) are now written in weak form. We introduce the following test functions:

- $\widetilde{n}^{\star} \in \mathscr{V}_{n}(\Omega)$ where $\mathscr{V}_{n}(\Omega)=\left\{\widetilde{n}^{\star} \mid \widetilde{n}^{\star} \in W^{1,2}(\Omega) \wedge \widetilde{n}\left(\Gamma_{n}\right)=0\right\}$

- $\widetilde{\boldsymbol{u}} \in \mathscr{V}_{u}(\Omega)$ where $\mathscr{V}_{u}(\Omega)=\left\{\widetilde{\boldsymbol{u}} \mid \tilde{\boldsymbol{u}} \in W^{1,2}(\Omega) \wedge \widetilde{u}_{i}\left(\Gamma_{u}\right)=0\right\}$

- $\tilde{\varphi} \in \mathscr{V}_{\varphi}(\Omega)$ where $\mathscr{V}_{\varphi}(\Omega)=\left\{\tilde{\varphi} \mid \widetilde{\varphi} \in W^{1,2}(\Omega) \wedge \widetilde{\varphi}\left(\Gamma_{\varphi}\right)=0\right\}$

where

$W^{m, p}(\Omega)=\left\{w \in L^{p}(\Omega)\left|D^{\alpha} w \in L^{p}(\Omega) \forall\right| \alpha \mid \leq m\right\}$ 


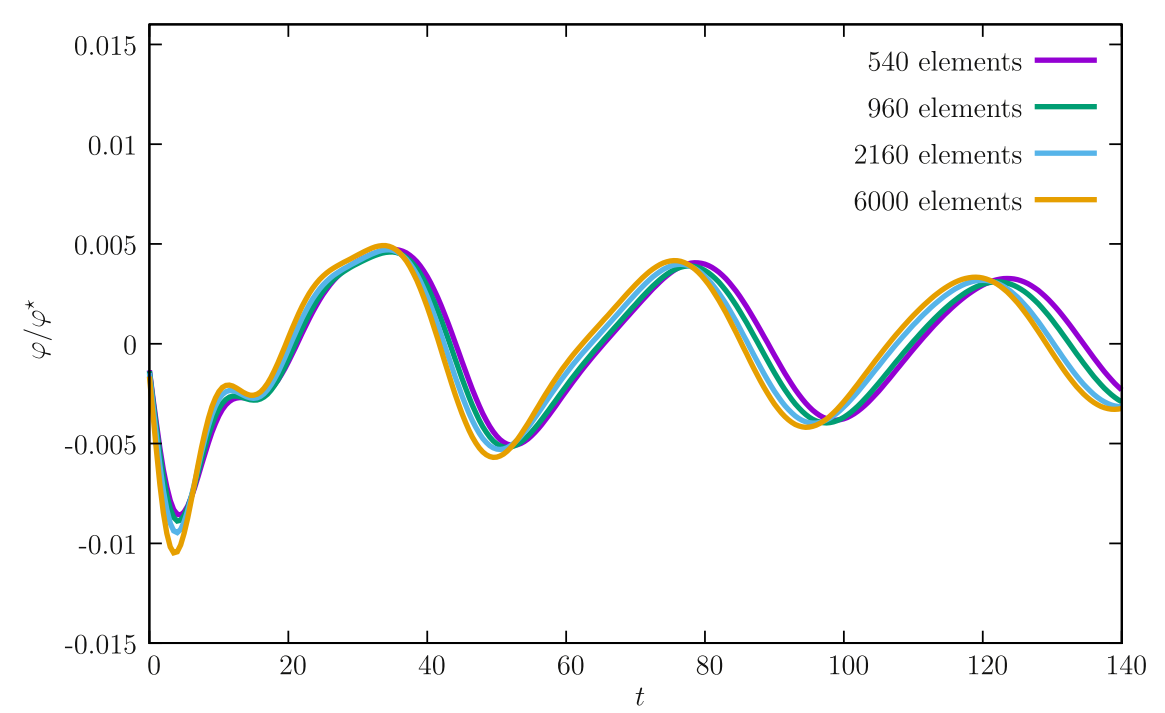

(a) Point A: evolution of $\varphi / \varphi^{\star}$ for four different meshes, $\Delta t=0.5 \mathrm{~s}$.

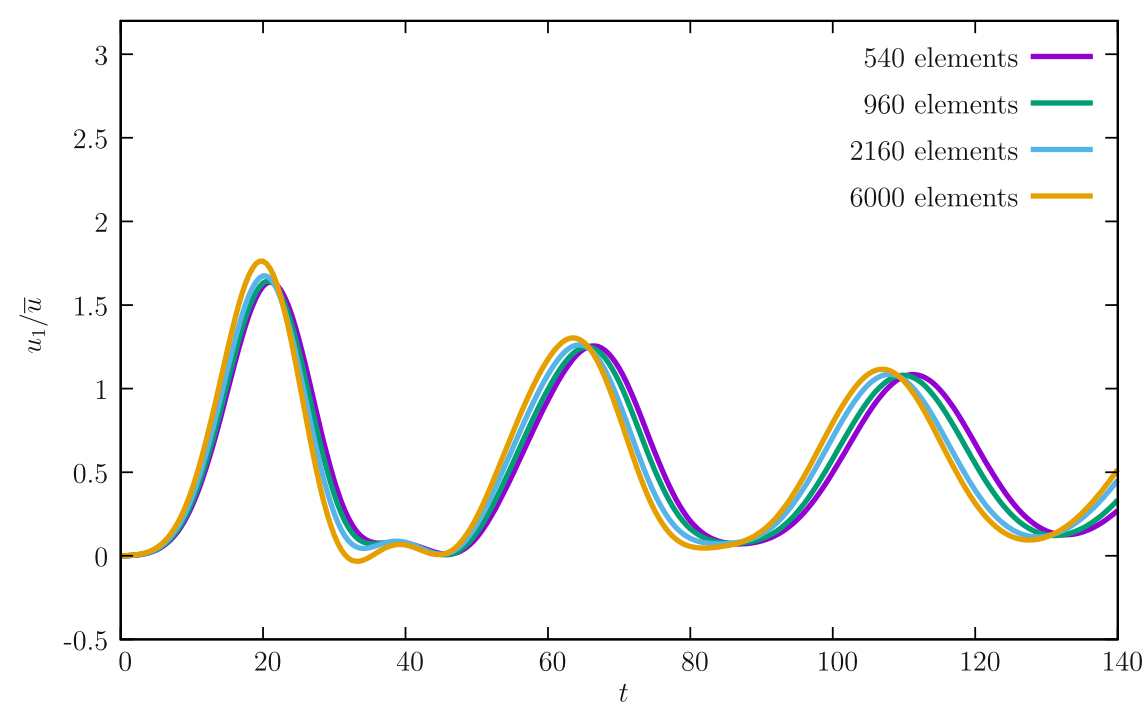

(b) Point B: evolution of $u_{1}$ for four different meshes, $\Delta t=0.5 \mathrm{~s}$.

Fig. 6. Variable width slab: relevant data and results for $u_{1}$ and $\varphi$. Points A and B indicate monitored $u_{1}$ and $\varphi / \varphi^{\star}$, respectively.

and $L^{p}(\Omega)$ is the space of $p$-power integrable functions. With this purpose, we use the test functions to obtain a weak form of (21-23) integrating in the domain $\Omega$, where $k$ and $k+1$ are the time-step indices and $j$ is the direction index. This non-symmetry in the test/trial functions was introduced by

$$
\begin{aligned}
& \widetilde{W}=\int_{\Omega} \widetilde{n}^{\star}\left[\left(n_{k+1}^{\star}+1\right) \frac{\partial u_{j}}{\partial x_{j}}+\frac{n_{k+1}^{\star}-n_{k}^{\star}}{\Delta t}+\frac{\partial n_{k+1}^{\star}}{\partial x_{j}} u_{j}\right] \mathrm{d} \Omega+\int_{\Omega} \widetilde{\boldsymbol{u}}^{\bullet T}\left[\left(n_{k+1}^{\star}+1\right)\left(\frac{\boldsymbol{u}_{k+1}-\boldsymbol{u}_{k}}{\Delta t}\right)+\left.\left(n_{k+1}^{\star}+1\right)\left\{\begin{array}{l}
u_{1} \frac{\partial u_{1}}{\partial x_{1}}+u_{2} \frac{\partial u_{1}}{\partial x_{2}} \\
u_{1} \frac{\partial u_{2}}{\partial x_{1}}+u_{2} \frac{\partial u_{2}}{\partial x_{2}}
\end{array}\right\}\right|_{k+1}\right]_{d} \\
& +\int_{\Omega} \widetilde{u}^{\bullet T}\left[-\left.\frac{e\left(n_{k+1}^{\star}+1\right) z_{d}}{m_{d}}\left\{\begin{array}{l}
\frac{\partial \varphi}{\partial x_{1}} \\
\frac{\partial \varphi}{\partial x_{2}}
\end{array}\right)\right|_{k+1}+\left.\int_{\Omega} \frac{c_{d a}^{2}}{n_{d 0}}\left\{\begin{array}{l}
\frac{\partial n^{\star}}{\partial x_{1}} \\
\frac{\partial n^{\star}}{\partial x_{2}}
\end{array}\right\}\right|_{k+1}\right] \mathrm{d} \Omega+\int_{\Omega}\left\{-\nabla \varphi_{k+1} \cdot \nabla \tilde{\varphi}+\widetilde{\varphi} \frac{e}{\varepsilon_{0}}\left[n_{i}-n_{e}-n_{d 0}\left(1+n_{k+1}^{\star}\right) z_{d}\right]\right\} \mathrm{d} \Omega \\
& +\int_{\Gamma_{\varphi^{\prime}}} \widetilde{\varphi} \underbrace{\nabla \varphi_{k+1} \cdot v}_{\tilde{t}(t)} \mathrm{d} \Gamma_{\varphi^{\prime}}=0
\end{aligned}
$$




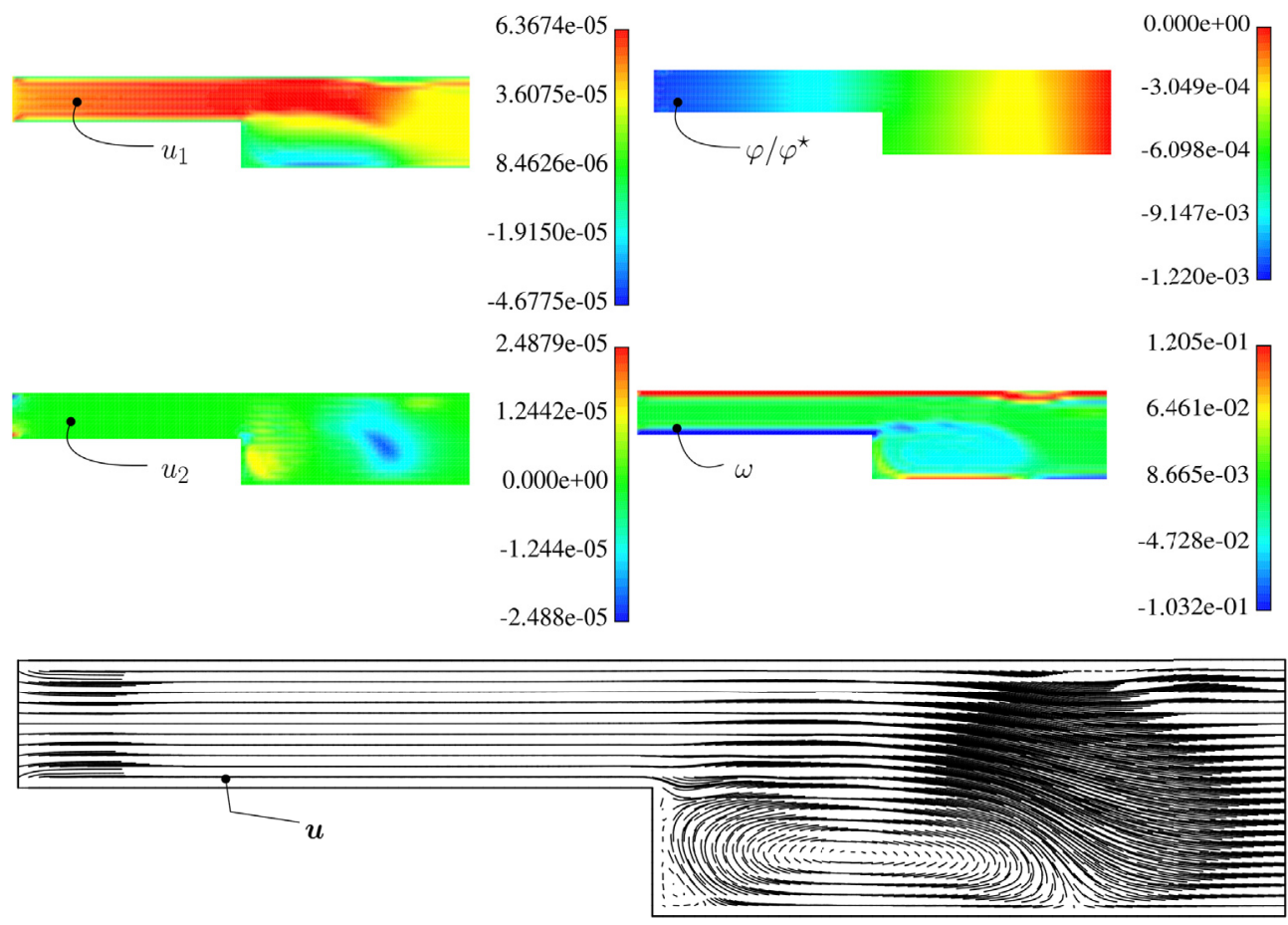

Fig. 7. Variable width slab $(t=500 \mathrm{~s})$ : contour plots for $u_{1}, u_{2}, \varphi$ and $\omega$. The velocity vectors are also shown.

Brooks and Hughes [20] to ensure stability in the presence of convection. We directly follow Zienkiewicz, et al. [21] in the present application. The specific form of the test function $\widetilde{\boldsymbol{u}}^{\bullet}$ will be detailed later. For the application of the Newton-Raphson method, we require the first variation of (25), for which we will make use of the symbol d:
We note that, in deriving (26), we made use of the property $\mathrm{d} \tilde{\boldsymbol{u}}=\mathbf{0}$. However, note that $\mathrm{d} \widetilde{\boldsymbol{u}}^{\bullet} \neq \mathbf{0}$. The Newton solution is based on the two definitions (25-26) using standard techniques (see, e.g. Ref. [22]).

$$
\begin{aligned}
& \mathrm{d} \widetilde{W}=\int_{\Omega} \widetilde{n}^{\star}\left[\mathrm{d} n_{k+1}^{\star} \frac{\partial u_{j}}{\partial x_{j}}+\left(n_{k+1}^{\star}+1\right) \frac{\partial \mathrm{d} u_{j}}{\partial x_{j}}+\frac{\mathrm{d} n_{k+1}^{\star}}{\Delta t}+\frac{\partial n_{k+1}^{\star}}{\partial x_{j}} \mathrm{~d} u_{j}+\frac{\partial \mathrm{d} n_{k+1}^{\star}}{\partial x_{j}} u_{j}\right] \mathrm{d} \Omega \\
& +\int_{\Omega} \tilde{\boldsymbol{u}}^{\bullet T}\left[\left(\frac{n_{k+1}^{\star}+1}{\Delta t}\right) \mathrm{d} \boldsymbol{u}_{k+1}+\mathrm{d} n_{k+1}^{\star}\left(\frac{\boldsymbol{u}_{k+1}-\boldsymbol{u}_{k}}{\Delta t}\right)+\left.\mathrm{d} n_{k+1}^{\star}\left\{\begin{array}{l}
u_{1} \frac{\partial u_{1}}{\partial x_{1}}+u_{2} \frac{\partial u_{1}}{\partial x_{2}} \\
u_{1} \frac{\partial u_{2}}{\partial x_{1}}+u_{2} \frac{\partial u_{2}}{\partial x_{2}}
\end{array}\right\}\right|_{k+1}\right] \mathrm{d} \Omega \\
& +\int_{\Omega} \widetilde{\boldsymbol{u}}^{\bullet T}\left(n_{k+1}^{\star}+1\right)\left\{\begin{array}{l}
\mathrm{d} u_{1} \frac{\partial u_{1}}{\partial x_{1}}+\mathrm{d} u_{2} \frac{\partial u_{1}}{\partial x_{2}}+u_{1} \frac{\partial \mathrm{d} u_{1}}{\partial x_{1}}+u_{2} \frac{\partial \mathrm{d} u_{1}}{x_{2}} \\
\mathrm{~d} u_{1} \frac{\partial u_{2}}{\partial x_{1}}+\mathrm{d} u_{2} \frac{\partial u_{2}}{\partial x_{2}}+u_{1} \frac{\partial \mathrm{d} u_{2}}{\partial x_{1}}+u_{2} \frac{\partial \mathrm{d} u_{2}}{\partial x_{2}}
\end{array}\right\} \mathrm{d} \Omega \\
& +\int_{\Omega} \tilde{\boldsymbol{u}}^{\bullet T}\left[-\left.\frac{e\left(n_{k+1}^{\star}+1\right) Z_{d}}{m_{d}}\left\{\begin{array}{l}
\frac{\partial \mathrm{d} \varphi}{\partial x_{1}} \\
\frac{\partial \mathrm{d} \varphi}{\partial x_{2}}
\end{array}\right\}\right|_{k+1}-\left.\frac{e Z_{d}}{m_{d}} \mathrm{~d} n_{k+1}^{\star}\left\{\begin{array}{l}
\frac{\partial \varphi}{\partial x_{1}} \\
\frac{\partial \varphi}{\partial x_{2}}
\end{array}\right\}\right|_{k+1}+\left.\int_{\Omega} \frac{c_{d a}^{2}}{n_{d 0}}\left\{\begin{array}{c}
\frac{\partial \mathrm{d} n^{\star}}{\partial x_{1}} \\
\frac{\partial \mathrm{d} n^{\star}}{\partial x_{2}}
\end{array}\right\}\right|_{k+1}\right] \mathrm{d} \Omega \\
& +\int_{\Omega} \mathrm{d} \widetilde{\boldsymbol{u}}^{\bullet T}\left[\left(n_{k+1}^{\star}+1\right)\left(\frac{\boldsymbol{u}_{k+1}-\boldsymbol{u}_{k}}{\Delta t}\right)+\left.\left(n_{k+1}^{\star}+1\right)\left\{\begin{array}{l}
u_{1} \frac{\partial u_{1}}{\partial x_{1}}+u_{2} \frac{\partial u_{1}}{\partial x_{2}} \\
u_{1} \frac{\partial u_{2}}{\partial x_{1}}+u_{2} \frac{\partial u_{2}}{\partial x_{2}}
\end{array}\right\}\right|_{k+1}\right] \mathrm{d} \Omega \\
& +\int_{\Omega} \mathrm{d} \widetilde{\boldsymbol{u}}^{\bullet T}\left[-\left.\frac{e\left(n_{k+1}^{\star}+1\right) Z_{d}}{m_{d}}\left\{\begin{array}{l}
\frac{\partial \varphi}{\partial x_{1}} \\
\frac{\partial \varphi}{\partial x_{2}}
\end{array}\right\}\right|_{k+1}+\left.\int_{\Omega} \frac{c_{d a}^{2}}{n_{d 0}}\left\{\begin{array}{l}
\frac{\partial n^{\star}}{\partial x_{1}} \\
\frac{\partial n^{\star}}{\partial x_{2}}
\end{array}\right\}\right|_{k+1}\right] \mathrm{d} \Omega \\
& +\int_{\Omega}\left\{-\nabla \mathrm{d} \varphi_{k+1} \cdot \nabla \widetilde{\varphi}+\widetilde{\varphi} \frac{e}{\varepsilon_{0}}\left[\frac{\mathrm{d} n_{i}}{\mathrm{~d} \varphi} \mathrm{d} \varphi-\frac{\mathrm{d} n_{e}}{\mathrm{~d} \varphi} \mathrm{d} \varphi-\mathrm{d} n_{k+1}^{\star} n_{d 0} Z_{d}\right]\right\} \mathrm{d} \Omega
\end{aligned}
$$



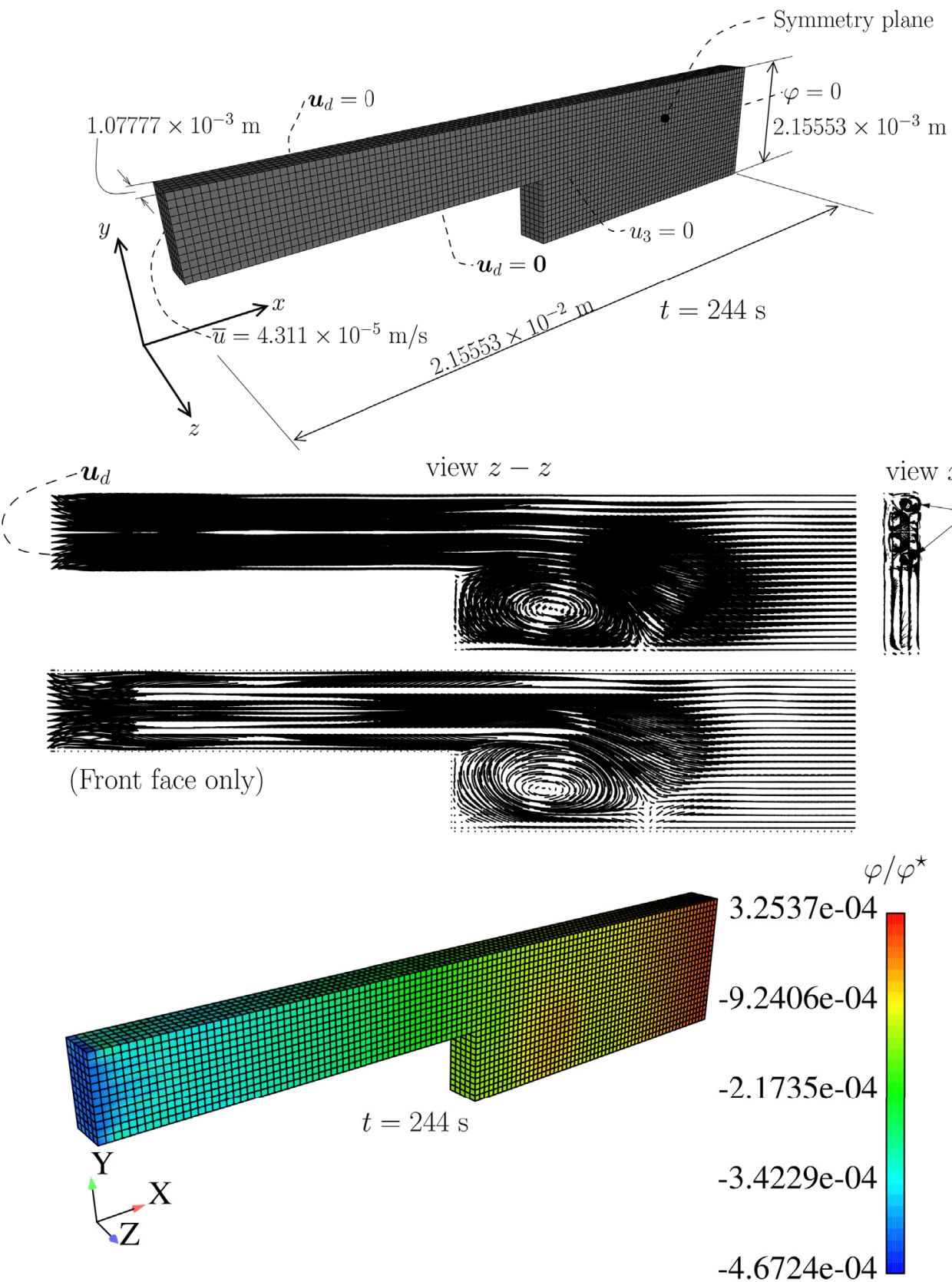

Fig. 8. 3D version of the variable width slab. With the addition of a symmetry plane, boundary conditions are similar to those of Fig. 6, but additional vortices occur at the inlet.

\section{Petrov-Galerkin discretization}

In terms of discretization, we introduce the interpolations for a given element $e$. Use is made of the interpolation matrices $\boldsymbol{N}_{e}(\boldsymbol{\xi})$ for $\boldsymbol{u}_{d}$ and $\mathbf{N}_{e}(\boldsymbol{\xi})$ for scalars $n^{\star}$ and $\varphi$, with $\boldsymbol{\xi}$ being the parent-domain coordinates. Introducing the nodal unknowns (trial functions) for a given element $e$ $\left(\boldsymbol{u}_{e}, n_{e}^{\star}\right.$, and $\left.\varphi_{e}\right)$, we have the interpolations (for simplicity we omit the subscript $d$ ):

$\boldsymbol{u}=\boldsymbol{N}_{e}(\boldsymbol{\xi}) \boldsymbol{u}_{e}$

$n^{\star}=\mathbf{N}_{e}(\xi) \boldsymbol{n}_{e}^{\star}$

$\widetilde{\varphi}=\mathbf{N}_{e}(\boldsymbol{\xi}) \tilde{\varphi}_{e}$

For the Galerkin projection (25) we require the test functions:

$\widetilde{\boldsymbol{u}}=\boldsymbol{N}_{e}(\boldsymbol{\xi}) \tilde{\boldsymbol{u}}_{e}$
$\widetilde{\boldsymbol{u}}^{\bullet}=\boldsymbol{N}_{e}^{\bullet}\left(\xi, \boldsymbol{u}_{e}\right) \widetilde{\boldsymbol{u}}_{e}$

$\tilde{n}^{\star}=\mathbf{N}_{e}(\xi) \tilde{\boldsymbol{n}}_{e}^{\star}$

$\widetilde{\varphi}=\mathbf{N}_{e}(\boldsymbol{\xi}) \widetilde{\varphi}_{e}$

where matrices $\mathbf{N}_{e}(\boldsymbol{\xi}), \boldsymbol{N}_{e}(\boldsymbol{\xi})$ and $\boldsymbol{N}_{e}^{\bullet}(\boldsymbol{\xi}, \boldsymbol{u})$ are defined as:

$\mathbf{N}_{e}(\xi)=\left[N_{1}(\xi) \cdots N_{n n e}(\xi)\right]$

$\boldsymbol{N}_{e}(\boldsymbol{\xi})=\left[\begin{array}{cccccc}N_{1}(\boldsymbol{\xi}) & 0 & N_{2}(\boldsymbol{\xi}) & \ldots & N_{\text {nne }}(\boldsymbol{\xi}) & 0 \\ 0 & N_{1}(\boldsymbol{\xi}) & 0 & N_{2}(\boldsymbol{\xi}) & \ldots & N_{\text {nne }}(\boldsymbol{\xi})\end{array}\right]$ 


$$
\begin{aligned}
& \boldsymbol{N}_{e}^{\bullet}(\boldsymbol{\xi}, \boldsymbol{u}) \\
& \quad=\left[\begin{array}{cccccc}
N_{1}^{\bullet}(\xi, \boldsymbol{u}) & 0 & N_{2}^{\bullet}(\boldsymbol{\xi}, \boldsymbol{u}) & \ldots & N_{\text {nne }}^{\bullet}(\boldsymbol{\xi}, \boldsymbol{u}) & 0 \\
0 & N_{1}^{\bullet}(\xi, \boldsymbol{u}) & 0 & N_{2}^{\bullet}(\boldsymbol{\xi}, \boldsymbol{u}) & \ldots & N_{n n e}^{\bullet}(\xi, \boldsymbol{u})
\end{array}\right]
\end{aligned}
$$

In definitions (34-36), nne is the number of nodes in each element, here taken as 4 for low-order quadrilateral and 8 for the hexahedron. $N_{K}^{\bullet}(\boldsymbol{\xi}, \boldsymbol{u})$ is given by (cf. [21])

$N_{K}^{\bullet}(\xi, \boldsymbol{u})=N_{K}(\xi)+\frac{\alpha h}{2} \frac{u_{i}}{\|\boldsymbol{u}\|} \frac{\partial N_{K}}{\partial x_{i}}$

where $\alpha$ is a stabilization parameter, and $h$ is the element characteristic length. The optimal value of $\alpha$ is given by Ref. [21]:

$\alpha=\operatorname{coth} P e-\frac{1}{P e}$

where the Péclet number is defined as

$P e=\|\boldsymbol{u}\| h / 2 k$

In (39), $k$ is the diffusion constant. Since the diffusion constant, $k$, is zero in the present case, we have $\alpha=1$. This corresponds to the Streaming Upwind Petrov-Galerkin (SUPG) $[19,21]$, specializing the element size from the velocity. The element characteristic length, $h$, is defined in agreement with Tezduyar and Park [23], as:

$h=\frac{2\|\boldsymbol{u}\|}{\sum_{K=1}^{n n e}\left|\frac{\partial N_{K}}{\partial X_{i}} u_{i}\right|}$

where $N_{K}$ are the shape functions and nne denotes the number of nodes in each element. We use the isoparametric interpolation [24]. For a low-order quadrilateral we have nne $=4$, and the shape functions have the following form:

$N_{K}(\xi)=\frac{1}{4}\left(1+\xi_{1} \xi_{1 K}\right)\left(1+\xi_{2} \xi_{2 K}\right)$

$\left\{\xi_{1 K}\right\}=\{-1,1,1,-1\}$

$\left\{\xi_{2 K}\right\}=\{-1,-1,1,1\}$

and, for the hexahedron,

$N_{K}(\xi)=\frac{1}{8}\left(1+\xi_{1} \xi_{1 K}\right)\left(1+\xi_{2} \xi_{2 K}\right)\left(1+\xi_{3} \xi_{3 K}\right)$

$\left\{\xi_{1 K}\right\}=\{-1,-1,-1,-1,+1,+1,+1,+1\}$

$\left\{\xi_{2 K}\right\}=\{-1,-1,+1,+1,-1,-1,+1,+1\}$

$\left\{\xi_{3 K}\right\}=\{-1,+1,-1,+1,-1,+1,-1,+1\}$

\section{Numerical examples}

We implemented two specific finite elements (quadrilateral and hexahedral) in SimPlas [26]. The ionosphere properties (cf. [25]) are adopted and shown in Table 2. We consider a spherical $\mathrm{Al}_{2} \mathrm{O}_{3}$ dust particle with a radius of $r_{d}=1 \times 10^{-6} \mathrm{~m}$ and $\rho_{d}=3950 \mathrm{Kg} / \mathrm{m}^{3}$. This agrees with the interval given in Ref. [25]. The first example deals with an initial density (and hence charge) imbalance with prescribed $\varphi$ at the boundary. After this preliminary test, a variable-width slab is presented, where a vortex occurs due to change in section for the 2D case and, in $3 \mathrm{D}$, additional vortices appear at the inlet region. With this example, a complete convergence study is performed.

\subsection{Square with initial charge imbalance}

We now analyze a square plate with initial imbalance of charge. Fig. 1 shows the relevant data, with the charge imbalance being imposed as an initial condition. The goal is to assess the mesh size effect on the results $\left(\varphi / \varphi^{\star}, \boldsymbol{u}_{d}=\left\{u_{1}, u_{2}\right\}\right.$ and $\left.n_{d}^{\star}\right)$. Two meshes are considered: $40 \times 40$ (coarse) and $60 \times 60$ (fine) square elements.

Nonlinearity is represented by two parameters, related to definitions (14-15):

$\mathrm{c}_{e}=\frac{\exp \left(\frac{e \varphi}{\kappa_{B} T_{e}}\right)}{1+\frac{e \varphi}{\kappa_{B} T_{e}}}-1$

$c_{i}=\frac{\exp \left(-\frac{e \varphi}{\kappa_{B} T_{i}}\right)}{1-\frac{e \varphi}{\kappa_{B} T_{i}}}-1$

When the problem is linear, $c_{e}=c_{i}=0$ and both (48-49) are subsequently used in contour plots to assess nonlinearities. Fig. 1 shows the contour plots for the aforementioned quantities for $t=10 \mathrm{~s}$. Smooth results are obtained, which are a consequence of the SUPG technique. Fig. 2 shows the comparison between the two meshes. It can be observed that results show slight differences for $t=2.5 \mathrm{~s}$. Nonlinearities can be observed in Fig. 1 where the two parameters $c_{e}$ and $c_{i}$ are plotted.

\subsection{Vortex in a variable width slab}

In this test, we introduce slab with a change of section in the direction of flow with the objective of testing the robustness of our combination Petrov-Galerkin/Backward Euler integration. Fig. 3 shows the dimensions and relevant data for this problem. Time-step sensitivity was found to be very good with the larger time step $\Delta t=0.5 \mathrm{~s}$ providing acceptable detail. In terms of mesh sensitivity, we test 4 meshes, all with square elements. Only slight dependence is observed, cf. Fig. 6. Contour plots for $u_{1}, u_{2}, \varphi$ and $\omega$ are shown in Fig. 7. We can observe the smooth behavior of all quantities.

We use a very fine mesh with 13500 elements as a reference solution to assess the mesh convergence of $u_{1} / \bar{u}$ and $\varphi / \varphi^{\star}$. Errors are defined using the uniform norm as:

$e_{u}=\frac{\left\|u_{1} / \bar{u}-u_{r e f} / \bar{u}\right\|_{\infty,[0,140]}}{\left\|u_{r e f} / \bar{u}\right\|_{\infty,[0,140]}}$

$e_{\varphi}=\frac{\left\|\varphi / \varphi^{\star}-\varphi_{r e f} / \varphi^{\star}\right\|_{\infty,[0,140]}}{\left\|\varphi_{r e f} / \varphi^{\star}\right\|_{\infty,[0,140]}}$

where the interval $t \in[0,140] \mathrm{s}$ is used. Fig. 4 shows $e_{u}$ and $e_{\varphi}$ as functions of $h$ where $h$ is the edge size of the square elements. We use 5 different values of $h$ to assess convergence: $h=3.59241 \times 10^{-4}, 2.69441 \times$ $10^{-4}, 1.34721 \times 10^{-4}, 1.19761 \times 10^{-4}, 1.07777 \times 10^{-4}$. We conclude that linear convergence occurs with this formulation.

Alternative time-integration algorithms exist for the fluid equations, one of the most popular is the Crank-Nicolson algorithm [27], which combines fully backward-Euler and forward-Euler terms to obtain second-order convergence. We found that it allows larger time-steps, at the cost of more function evaluations, see Fig. 5. Therefore, for relatively inexpensive function evaluations, the Crank-Nicolson algorithm is preferable.

The 3D version of the slab is shown in Fig. 8, where the boundary conditions are equivalent to those in Fig. 3, but with the zero velocity around the external boundary, except in the symmetry plane, in which $u_{3}=0$. Fig. 8 shows another type of vortex, which was found in the inlet section, with a rotation axis parallel to $x$. The vortex after the jump is also shown in this Figure and is similar to what was found in the 2D example. In the view $z-z$ we can see the variation of the vortex shape at the jump along the thickness (the front face alone is shown below). 


\section{Conclusions}

We introduced a new formulation for dust-acoustic waves in unmagnetized plasma. This consists of a Petrov-Galerkin finite element combined with the backward-Euler time integration method. Nonlinear effects are included in the number density of ions and electrons, which are affected exponentially by the electrostatic potential. Numerical experiments showed very good robustness with respect to mesh size and time-step size and absence of instabilities. Waves resulting from the nonlinear constitutive laws for number densities are sharply observed. As a follow-up contribution, we will include viscosity (less important than in classical plasma analysis, cf. [11]) and will introduce the magnetic field, along with a specialized version of Maxwell's equations.

\section{References}

[1] I. Langmuir, Oscillations in ionized gases, Proc. Natl. Acad. Sci. 14 (August 1928) 627-637.

[2] F.F. Chen, Introduction to Plasma Physics and Controlled Fusion, 2 edition, Plasma Physics, vol. 1, Plenum Press, New York, 1984.

[3] P.K. Shukla, A.A. Mamun, Introduction to Dusty Plasma Physics. Series in Plasma Physics, IOP Institute of Physics Publishing, 2002.

[4] C.K. Goertz, G. Morfill, A model for the formation of spokes in Saturn's ring, Icarus 53 (1983) 219-229.

[5] M. Horanyi, H.L.F. Houpis, D.A. Mendis, Charged dust in the earth's magnetosphere, Astrophys. Space Sci. 144 (1-2) (1988) 215-229.

[6] O. Havnes, C.K. Goertz, G.E. Morfill, E. Grün, W. Ip, Dust charges, cloud potential, and instabilities in a dust cloud embedded in a plasma, J. Geophys. Res. 92 (A3) (1987) 2281-2287.

[7] N.N. Rao, P.K. Shukla, M.Y. Yu, Dust-acoustic waves in dusty plasmas, Planet Space Sci. 38 (4) (1990) 543-546.

[8] F.G. Verheest, General dispersion relations for linear waves in multicomponent plasmas, Physica 34 (1967) 17-35.

[9] A.A. Mamun, P.K. Shukla, Electrostatic solitary and shock structures in dusty plasmas, Phys. Scr. 98 (2002) 107-114.

[10] A. Barkan, R.L. Merlino, N. D'Angelo, Laboratory observation of dust-acoustic wave mode, Phys. Plasmas 2 (10) (1995) 3563-3565.
[11] R. Merlino, J. Goree, Dusty plasmas in the laboratory, industry, and space, Phys. Today 57 (7) (2004) 32-38.

[12] C. Sovinec, A. Glasser, T. Gianakon, D. Barnes, R. Nebel, S. Kruger, D. Schnack, S Plimpton, A. Tarditi, M. Chu, Nonlinear magnetohydrodynamics simulation using high-order finite elements, J. Comput. Phys. 195 (1) (2004) 355-386.

[13] C.R. Sovinec, J.R. King, Analysis of a mixed semi-implicit/implicit algorithm for low-frequency two-fluid plasma modeling, J. Comput. Phys. 229 (2010) 5803-5819.

[14] S.C. Jardin, J. Breslau, N. Ferraro, A high-order implicit finite element method for integrating the two-fluid magnetohydrodynamic, J. Comput. Phys. 226 (2007) 2146-2174.

[15] R. Codina, N. Hernández-Silva, Stabilized finite element approximation magneto-hydrodynamics equations, Comput. Mech. 38 (2006) 344-355.

[16] J.N. Shadid, R.P. Pawlowski, E.C. Cyr, R.S. Tuminaro, L. Chacón, Scalable implicit incompressible resistive MHD with stabilized FE and fully-coupled Newton-Krylov-AMG, Comp. Method Appl. M. 304 (2016) 1-25.

[17] B. Srinivasan, A. Hakim, U. Shumlak, Numerical methods for two-fluid dispersive fast MHD phenomena, Commun. Comput. Phys. 10 (1) (2011) 183-215.

[18] D. Levy, C.-W. Shu, J. Yan, Local discontinuous galerkin methods for nonlinear dispersive equations, J. Comput. Phys. 196 (2004) 751-772.

[19] T.J.R. Hughes, A.N. Brooks, A multi-dimensional upwind scheme with no cross wind diffusion, in: T.J.R. Hughes (Ed.), Finite Elements for Convection Dominated Flows, Number 34, ASME, AMD, New York, 1979.

[20] A.N. Brooks, T.J.R. Hughes, Streamline upwind/Petrov-Galerkin formulations for convection dominated flows with particular emphasis on the incompressible Navier-Stokes equations, Comp. Method Appl. M. 32 (1982) 199-259.

[21] O.C. Zienkiewicz, R.L. Taylor, P. Nithiarasu, 7 edition, The Finite Element Method for Fluid Dynamics, vol. 3, Butterworth-Heinemann, Elsevier, 2014.

[22] K.-J. Bathe, Finite Element Procedures, Prentice-Hall, Englewood Cliffs, 1996.

[23] T.E. Tezduyar, Y.J. Park, Discontinuity capturing finite element formulations for nonlinear convection-diffusion-reaction problems, Comp. Method Appl. M. 59 (1986) 307-325.

[24] T.J.R. Hughes, The Finite Element Method, Linear Static and Dynamic Finite Element Analyses, Prentice-Hall, Englewood Cliffs, N.J, 1987.

[25] H. Fu, W.A. Scales, Model for charged dust expansion, Phys. Plasmas 20 (073704) (2013).

[26] P. Areias. Simplas. http://www.simplas-software.com.

[27] J. Crank, P. Nicolson, A practical method for numerical evaluation of solutions of partial differential equations of the heat-conduction type, Adv. Comput. Math. 6 (207-226) (1996) Reprinted from Proc Camb Phil Soc 43 (1947) 50-67. 